\title{
Evaluation of actin and desmin expression by immunohistochemistry to determine the time since death in dogs: A pilot study
}

\begin{abstract}
The estimation of the time since death can be of vital importance in a forensic investigation. Several different methods are available to the forensic investigator for estimation of time since death, including temperature- and molecular-based methods, and entomology. The use of immunohistochemical (IHC) methods has rarely been studied for estimation of time since death. To determine whether IHC staining can be used for estimation of time since death in dogs, we selected skeletal muscle as it is abundantly available and slower to decay compared to other tissues. Euthanized dogs (Canis familiaris) were allowed to decompose for up to 30 days in a walk-in refrigeration unit. Thigh muscle tissue was collected from a single dog on postmortem days $0,2,4,6, \ldots, 28,30$. Tissues were formalin-fixed and IHC-stained for muscle-specific actin and desmin. IHC staining was evaluated using the immunoreactive score (IRS) system. Skeletal muscle from all dogs stained positive for the duration of this study. Desmin staining was negative at days 10 and 26. The results show that immunohistochemistry can be performed on dog skeletal muscle for at least 30 days postmortem and support the further investigation of the use of immunohistochemistry for muscle markers to investigate the time since death for periods longer than 30 days.
\end{abstract}

KEYWORDS: veterinary forensic sciences, veterinary pathology, immunohistochemistry, dog, desmin, muscle-specific actin, time since death, postmortem interval, IHC

The estimation of time since death can be of critical importance in forensic investigations of humans and animals. It can answer the question, "How long was the victim deceased?" Thus, it can help to determine when the crime was committed. Knowing when the crime occurred allows investigators to include or exclude individuals from a pool of suspects (Brooks 2016).

Numerous methods can be used to estimate the time since death, including temperature- and molecular-based methods, postmortem chemistry (i.e., vitreous humor analysis), intraocular pressure changes, entomology, to name a few. However, no single method can be used reliably to estimate the time since death accurately (Brooks 2016). Microscopic methods also have been used for determining the time since death and include evaluation of histologic changes of human skin, testicular changes in the rat, and evaluation of a set of tissues in the dog (Bardale et al. 2012, Bryant and Boekelheide 2007, Erlandsson and Munro 2007). Immunohistochemical (IHC) staining of the pancreas and thyroid glands has been used as a method to estimate the time since death (Ortmann et al. 2017, Wehner et al. 1999, Wehner et al. 2000, Wehner et al. 2001a, Wehner et al. 2001b).

Positive results have been obtained from IHC staining performed on tissues taken at autopsy from individuals who were deceased for 7 to 14 days, and select markers remained positive greater than 14 days after death (Lesnikova et al. 2018). Although IHC staining is commonly utilized in veterinary diagnostics for the identification of infectious agents, determination of cell lineage, and prognostic purposes, its use for estimation of time since death has not been adequately assessed. A single report evaluated IHC staining for CD3 (T-cell marker) and CD79a (B- cell marker) of canine lymph nodes for the determination of time since death (Erlandsson and Munro 2007). The theory behind the use of immunohistochemistry for the determination of time since death is that an antibody will fail to bind to the associated antigen as the antigen undergoes postmortem change.

The purpose of this study was to evaluate if postmortem immunohistochemistry of dog (Canis familiaris) skeletal muscle can be used to assess time since death.

\section{Materials and Methods}

\section{Animals}

The study used 16 humanely euthanized dogs that were obtained from an animal control facility, all of which were euthanized for reasons unrelated to this study. Euthanasia was performed with an overdose of barbiturate by the animal control facility. The use of the dogs for this study was approved by the Institutional Animal Care and Use Committee (IACUC protocol no. 201810402). Each cadaver was placed in right lateral recumbency in a walk-in refrigeration unit. The temperature range within the refrigeration unit was 6.1 to $13.7^{\circ} \mathrm{C}$. Each dog was allowed to decompose in the refrigeration unit for at most 30 days. Skeletal muscle tissue from the lateral aspect of the right thigh was collected from a single dog on postmortem days $0,2,4$, $6, \ldots, 28,30$. Each dog was sampled only once throughout the study. Collected tissue, measuring approximately $3 \times 2 \times 1 \mathrm{~cm}$, was placed in $10 \%$ neutral-buffered formalin and was trimmed

\footnotetext{
1 Department of Comparative, Diagnostic, and Population Medicine, University of Florida College of Veterinary Medicine, PO Box 100123, Gainesville, FL 32610-0123, USA 
within 24 to 48 hours. Tissues were embedded in paraffin using standard procedures.

\section{Tissue microarrays}

Tissue microarrays were constructed by standard procedures. A 4-mm tissue punch was removed from each donor block and transferred into the recipient block. Recipient blocks were created using a commercially available mold (Newcomer Supply, Middleton, WI) (Figure 1). The recipient block was arranged according to a defined $\mathrm{x}-\mathrm{y}$ coordinate position. Lung and liver tissue cores from an unrelated block of dog tissue were used as position markers at one corner of each tissue microarray. After construction, the tissue microarray blocks were heated in an oven at $62{ }^{\circ} \mathrm{C}$ for 20 minutes. Paraffin was added to the block and subsequently frozen. All blocks were cut into $3-\mu \mathrm{m}$ thick sections using standard procedures.

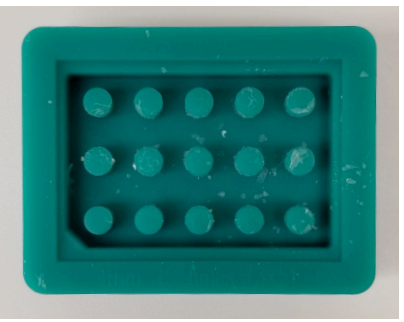

FIGURE 1-Commercially available mold used to make recipient blocks for tissue microarrays

\section{Immunohistochemistry}

The tissue microarrays were stained for muscle-specific actin (Biocare Medical, Pacheco, CA) and desmin (Novocastra, Buffalo Grove, IL). Canine small intestine from a dog unrelated to this study was used as the positive control for muscle-specific actin and desmin. All samples and corresponding positive controls were processed using the Leica Bond RX automated stainer (Leica Biosystems, Buffalo Grove, IL). Muscle-specific actin is a monoclonal mouse antibody (clone HHF35) in dilution 1/100. Muscle-specific actin antibody retrieval was done using a citrate buffer ( $\mathrm{pH}$ 6.0) for 20 minutes at $95^{\circ} \mathrm{C}$. Desmin was a monoclonal mouse antibody (clone DE-R-11) in dilution 1/100. Desmin antibody retrieval was done using a citrate buffer ( $\mathrm{pH}$ 6.0) for 20 minutes at $95^{\circ} \mathrm{C}$.

IHC stains were graded using the immunoreactive score (IRS) system (Fedchenko and Reifenrath 2014). Both muscle-specific actin and desmin IHC stains have cytoplasmic staining. IRSs were considered positive if the score was 2 to 3 (mildly positive), 4 to 8 (moderately positive), and 9 to 12 (strongly positive) (Table 1 ).

TABLE 1-Immunoreactive score (IRS) system for characterization of immunoreactivity for desmin and muscle-specific actin

\begin{tabular}{|c|c|c|}
\hline A, $\%$ of positive cells & B, intensity of staining & $\mathrm{IRS}=\mathrm{A} \times \mathrm{B}$ \\
\hline $0,0 \%$ & 0 , no reaction & $0-1$, negative \\
\hline $1,<10 \%$ & 1, mild reaction & 2-3, mildly positive \\
\hline $2,10-50 \%$ & $\mathbf{2}$, moderate reaction & 4-8, moderately positive \\
\hline $3,51-80 \%$ & 3 , intense reaction & $9-12$, strongly positive \\
\hline $4,>80 \%$ & & \\
\hline
\end{tabular}

\section{Results}

IHC staining for muscle-specific actin was observed in all simultaneously performed control samples and 16 specimens evaluated (Figure 2a-b). IHC staining for desmin was observed in all simultaneously performed control samples and 16 specimens evaluated (Figure $2 \mathrm{c}-\mathrm{d}$ ). The skeletal muscle had positive cytoplasmic immunoreactivity in 14 specimens evaluated, with specimens from days 10 and 26 being negative. Strongly positive cytoplasmic immunoreactivity for muscle-specific actin was observed on days $0,2,4$, and 24 ; and for desmin, on day 0 only (Table 2).

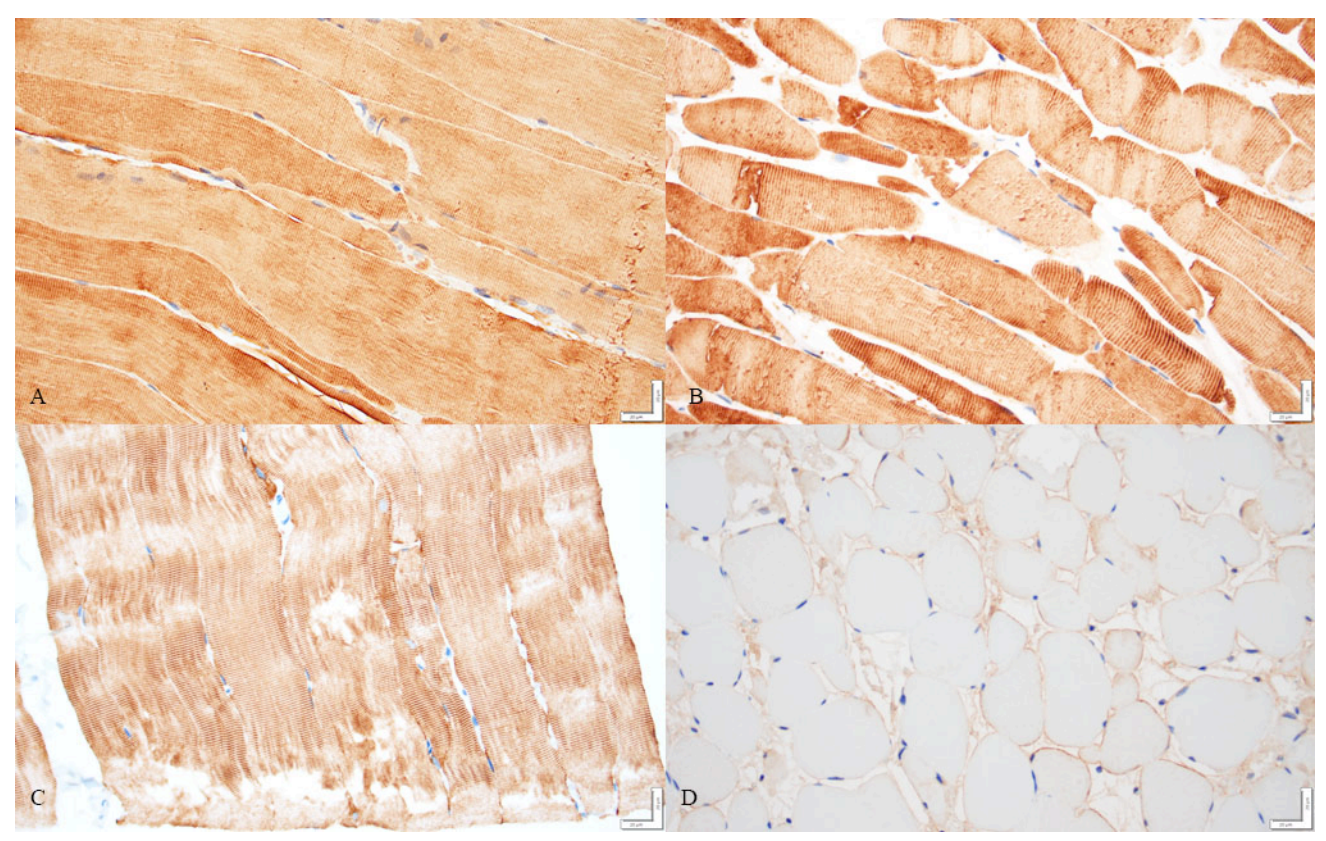

FIGURE 2-Cytoplasmic immunoreactivity in dog skeletal muscle. a) Muscle-specific actin, day 0 , positive; b) muscle-specific actin, day 30, positive; c) desmin, day 0 , positive; d) desmin, day 26, negative. $400 \times$. 
TABLE 2-Immunoreactive scores (IRSs) for desmin and muscle-specific actin in dog skeletal muscle

\begin{tabular}{lll}
\hline & & IRS \\
\cline { 2 - 3 } Day & Desmin & Muscle-specific actin \\
\hline 0 & strongly positive & strongly positive \\
2 & moderately positive & strongly positive \\
4 & moderately positive & strongly positive \\
6 & moderately positive & moderately positive \\
8 & mildly positive & moderately positive \\
10 & negative & mildly positive \\
12 & moderately positive & moderately positive \\
14 & moderately positive & moderately positive \\
16 & moderately positive & moderately positive \\
18 & moderately positive & moderately positive \\
20 & moderately positive & moderately positive \\
22 & moderately positive & moderately positive \\
24 & moderately positive & strongly positive \\
26 & negative & moderately positive \\
28 & moderately positive & moderately positive \\
30 & mildly positive & moderately positive \\
\hline
\end{tabular}

\section{Discussion}

Information regarding the use of immunohistochemistry for estimation of the time since death is limited. In humans, studies are limited to the thyroid gland (thyroglobulin, calcitonin) and pancreas (insulin, glucagon) (Ortmann et al. 2017, Wehner et al. 1999, Wehner et al. 2000, Wehner et al. 2001a, Wehner et al. 2001b). In humans, staining the thyroid gland for calcitonin revealed that c-cells were positive in all cases up to 4 days postmortem and negative in all cases after 13 days postmortem and between days 4 and 13 a mix of positive and negative results were observed (Wehner et al. 2001a). These results suggest that a deceased person with a negative result for calcitonin was deceased at least 5 days, whereas a positive result for calcitonin means the person was deceased for less than 12 days.

Skeletal muscle in Wistar rats has the most prolonged delay in postmortem change compared to other visceral organs, including the kidney, liver, pancreas, and heart (Tomita et al. 2004). Muscle protein degradation in humans has been shown to be a predictable process and associated with temperature and the time since death (Pittner et al. 2016). Porcine muscle (biceps femoris) was found to exhibit changes in protein expression, including desmin, calpain 1, and calpain 2 specific for particular time points after death (Pittner et al. 2016). Given the abundance and the delay in postmortem change, skeletal muscle serves as a possible tissue to assess a more prolonged time since death.

In the current study, we chose muscle-specific actin and desmin as candidate stains as these IHC stains are commonly performed in veterinary diagnostic laboratories. It was initially hypothesized that IHC staining would have become negative by 30 days postmortem due to the effects of decomposition on these antigens. Skeletal muscle maintained positive staining for musclespecific actin at all 16-time points with strongly positive staining on days $0,2,4$, and 24 . Staining for desmin was strongly positive on day 0 only. Negative staining for desmin was observed on days 10 and 26 in contrast to muscle-specific actin, where no negative staining was observed. The cause for the two isolated negative results for desmin is not known, although the staining technique is not suspected to be the cause since all tissues in the microarray undergo the same staining conditions. It is suspected that a variable(s) specific to the dogs was a factor (such as $\mathrm{pH}$ of muscle, more rapid protein denaturation, altered enzyme activity).

Interestingly, there also was a mildly positive IRS on day 10 for muscle-specific actin, suggestive that the unknown variable did not only affected the desmin IHC. The mixed positive immunoreactivity results for desmin and muscle-specific actin support the use of a panel of stains rather than a single stain in order to avoid a false negative. Overall, strongly positive scores were observed during the early collection points of this study, with moderately positive scores for much of the remainder of the study. Individual variation is suspected of having played a role in the strongly positive IRS score for muscle-specific actin from the day $24 \mathrm{dog}$. Many variables, both animal and environmental, can play a role in the rate of decomposition, and not all variables could be controlled in this study. In this study, the environmental factor of temperature was controlled and dogs of similar size were used. Similar to other studies that collected, stored, and handled specimens under the same conditions, there were variations of the results. According to one study, other factors to consider for individual patient variation include age, cause of death, medications and medical interventions, and overall health at the time of death (Blair et al. 2016). In this study, exact dog ages were not known, but life-stages appeared to range from young-adult to senior. All dogs had unknown medical histories. We could not control for other variables at the cellular level, such as variation in muscle $\mathrm{pH}$ and enzymatic activity, both of which could have led to variations in the IHC results.

Overall, it is shown that IHC could be used to evaluate the time since death for periods longer than 30 days when the body is refrigerated, and further studies are warranted. Since this was a pilot study, several inherent weaknesses exist. A notable weakness of this study was that each dog had muscle collected only once at a single time point. Future studies should evaluate multiple muscle specimens collected at multiple time points and from multiple sites (e.g., thigh, shoulder, head). This change in methodology would allow for improved evaluation of staining variations observed in individual dogs. Additional weaknesses were the small study population and lack of assessment of the effect of other environmental conditions (burial, submersion in water, or ground surface) on the immunoreactivity of these muscle markers. Some of these environmental conditions may be regionally specific; studies would have to be taken to assess for these differences. Given the association between muscle protein degradation with temperature and time since death, it can be hypothesized that if environmental conditions are changed, this will affect the staining pattern of the skeletal muscle.

\section{Conclusions}

Since no single method can be reliably used to determine the time since death accurately, exploration of novel methods to make this determination should be performed. As these methods are 
developed, they can be used in conjunction with other validated methods to provide more data for the estimation of time since death. In this study, it was shown that IHC staining for musclespecific actin and desmin could be performed on skeletal muscle from dogs that have been deceased for at least 30 days with positive staining results. As this is a pilot study, not enough testing was performed to recommend this method for use in its current form; however, preliminary results support further investigation for the use of muscle-specific actin and desmin as possible markers for estimation of time since death in cases with a more prolonged ( $>30$ days) postmortem interval. This pilot study used two skeletal muscle markers, and it is suspected that the use of additional muscle markers, such as myoglobin, troponin I, and skeletal-muscle myosin, might provide more information regarding the use of this modality to estimate the time since death. Lastly, future studies that include evaluating the time since death and IHC staining with muscle markers should be performed with varied environmental conditions and for more extended periods since death.

\section{Acknowledgments}

The author would like to Katrina Leser for assistance with tissue collection and Melissa Brown of the University of Florida Veterinary Diagnostic Laboratories for assistance with the IHC staining.

\section{Disclosures}

This research did not receive any specific grant from funding agencies in the public, commercial, or not-for-profit sectors.

\section{References}

Bardale RV, Tumram NK, Dixit PG, Deshmukh AY. 2012. Evaluation of histologic changes of the skin in postmortem period. Am J Forensic Med Pathol. 33:357-61.

Blair JA, Wang C, Hernandez D, Sidewalk SL, Rodgers MS, Achar RK, Fahey LM, Torres SL, Petersen RB, Zhu X, Casadesus G, Lee HG. 2016. Individual case analysis of postmortem interval time on brain tissue preservation. PLOS ONE. 11:e0151615.

Brooks JW. 2016. Postmortem changes in animal carcasses and estimation of the postmortem interval. Vet Pathol. 53:929-40.

Bryant BH, Boekelheide K. 2007. Time-dependent changes in post-mortem testis histopathology in the rat. Toxicol Pathol. 35: 665-71.

Erlandsson M, Munro R. 2007. Estimation of the post-mortem interval in beagle dogs. Sci Justice. 47:150-4.

Fedchenko N, Reifenrath J. 2014. Different approaches for interpretation and reporting of immunohistochemistry analysis results in the bone tissue - a review. Diagn Pathol. 9.

Lesnikova I, Schreckenbach MN, Kristensen MP, Papanikolaou LL, HamiltonDutoit S. 2018. Usability of immunohistochemistry in forensic samples with varying decomposition. Am J Forensic Med Pathol. 39:185-91.

Ortmann J, Dobererentz E, Madea B. 2017. Immunohistochemical methods as an aid in estimating time since death. Forensic Sci Int. 273:71-9.

Pittner S, Ehrenfellner B, Monticelli FC, Zissler A, Sänger AM, Stoiber W, Steinbacher P. 2016. Postmortem muscle protein degradation in humans as a tool for PMI delimitation. Int J Legal Med. 130:1547-55.

Ramos-Vera JA, Miller MA. 2014. When tissue antigens and antibodies get along: revisiting the technical aspects of immunohistochemistry-the red, brown, and blue technique. Vet Pathol. 51:42-87.

Tomita Y, Nihira M, Ohno Y, Sato S. 2004. Ultrastructural changes during in situ early postmortem autolysis in kidney, pancreas, liver, heart and skeletal muscle of rats. Legal Med. 6:25-31.

Wehner F, Wehner HD, Schieffer MC, Subke J. 1999. Delimitation of the time since death by immunohistochemical detection of insulin in pancreatic B-cells. Forensic Sci Int. 105:161-9.

Wehner F, Wehner HD, Schieffer MC, Subke J. 2000. Delimitation of the time since death by immunohistochemical detection of thyroglobulin. Forensic Sci Int. 110:199-206.

Wehner F, Wehner HD, Schieffer MC, Subke J. 2001a. Delimitation of the time since death by immunohistochemical detection of calcitonin. Forensic Sci Int. 122:9-94.

Wehner F, Wehner HD, Subke J. 2001b. Delimitation of the time since death by immunohistochemical detection of glucagon in pancreatic a-cells. Forensic Sci Int. 124:192-9. 